\title{
36 A PRACTICAL REPRESENTATION OF HOLONIC MANUFACTURING SYSTEMS
}

\author{
Shahin Rahimifard \\ Wolfson School of Mechanical and Manufacturing Engineering \\ Loughborough University, United Kingdom \\ S.Rahimifard@lboro.ac.uk
}

\begin{abstract}
Holonic manufacturing systems is now a well established research area with a significant body of work being undertaken which has resulted in generation of a large number of holonic manufacturing system configurations, architectures and reference models, together with various holonic approaches to the shop floor control, production scheduling, and machine control. There is now a common belief amongst the research community that the application of such holonic concepts would result in meeting the responsiveness and agility of manufacturing activities, necessitated by the modern pressures of the global market. However, the most important challenge remains the persuasion of decision makers within manufacturing companies and in particular small to medium enterprises to adopt these research results in their daily operation. The work reported in this paper is directed at addressing this challenge through realisation of a practical representation of holonic concepts to support a typical machining facility in a form of a laboratory-based demonstrator. In this practical presentation a conscious effort has been made to utilise commercially available and commonly used manufacturing software packages.
\end{abstract}

\section{INTRODUCTION}

The term holon was used initially by Koestler (1967) to describe an identifiable part of a system that possesses an individual identity and comprises of sub-ordinate parts. Another concept which Koestler introduced was that of a "holarchy", which is a stable, self-contained system consisting of holons as building blocks (Leeuwen and Norrie 1997). These concepts were adapted and applied to manufacturing systems by Suda (1990) and the Intelligent Manufacturing Systems (IMS) TC5 program to form the contemporary paradigm known as Holonic Manufacturing Systems (Matthews 1995, Van Brussel et al. 1996). In this contemporary paradigm, holons aim at both the co-operation with peer holons to achieve global manufacturing goals and also act autonomously as self-reliant units.

A number of interpretations of holonic concepts for manufacturing may be encountered in literature, and in recent years there has been a significant build up of holonic research initiatives. The most relevant of these to research reported in this paper are the use of holonic concepts in production planning (Sugimura and Moriwaki 1996, Gou et al. 1998, Sousa and Ramos 1999), shop floor control 
(Tonshoff and Winkler 1996, Balasubramanian et al. 2000), and developing intelligent controllers for manufacturing workstations (Wyns et al. 1996, Tanaya et al. 1997, Shin and Cho 2001). Furthermore, a number of researchers including the author have explored the application of holonic concepts in human centred systems within Small to Medium Enterprises (SMEs) as outlined in this paper. The research has investigated an innovative approach to management and control of manufacturing SMEs based on a conglomerate of distributed and autonomous holons which operate as a set of cooperating entities based on holonic concepts. In this paper the development of a comprehensive laboratory-based demonstrator is outlined based on implementation of three areas of research, namely a holonic information network, a holonic production planning and control structure and holonic manufacturing workstations. It is intended to utilise this holonic manufacturing demonstrator for $(a)$ industrial illustration and visualisation of complex issues related to an autonomous co-operative working practices within a distributed system and how such practices improve the flexibility and performance of manufacturing activities, and $(b)$ the training of managers and production operators to be able to adopt this new working culture.

\section{HOLONIC REPRESENTATION OF AN SME}

SMEs represent the largest proportion of the manufacturing sector, generating more than half of the total production output, in every industrial country. The production system of SMEs are typified by human centred manufacturing systems where the flexibility introduced through utilisation of often multi-skilled operators plays a major role in the agile performance and responsiveness of the company. The major assertions made in this paper are: that the familiar information system hierarchy and range of IT tools employed in larger companies are inappropriate for SMEs. In pursuit of realisation of more efficient IT tools for SMEs a new innovative approach is adopted based on groups of distributed and autonomous holons, operating in a collaborative manner. A holonic business model is developed to represent activities of a typical manufacturing SME, as depicted in figure 1. This model represents an enterprise as an enhanced organisation holarchy which can be viewed to consists of three main holons namely: the executive holon that represents the ultimate decisionmaking process within the company; the business holon that covers administration activities such as order processing, finance, costing, process planning and scheduling etc.; and the manufacturing holon involving the implementation and monitoring of the production plans produced by the business holon.

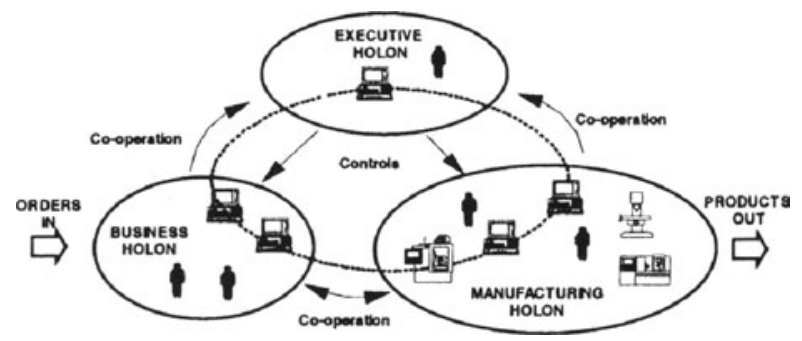

Figure 1 - The Conceptual Holonic Representation of an IT Supported SME 
Due to the vital role of individual operators within SMEs, the research is committed to realising the information and planning systems that support the human activity rather than enveloping them. The research was structured around three major work streams as outline below.

\subsection{The Generation of a Holonic Information System.}

The goal for the specification and design of the holonic information system was to reduce all information subsystems into a one homogeneous integrated system. As the basis for the development of such a homogenous information network, a SME enterprise model is constructed. To capture the holonic perspective within this SME enterprise reference model, a novel three phase approach of control, co-operation and autonomy has been utilised. Readers are referred to Toh et al. 1998 for more detailed information on this novel three phase approach which not only enables the information requirements to be modelled but also allows the human centred behaviour of the SME organisation to be represented. The realisation of a holonic information system (HIS) based on this SME enterprise model comprises of a number of node holons, which are defined as a composite of human, manufacturing equipment or software systems and their IT interfaces is depicted in figure 2 . The HIS network is supported by a manufacturing and an order database which store and maintain data required to facilitate the functionality of such homogenous information system.

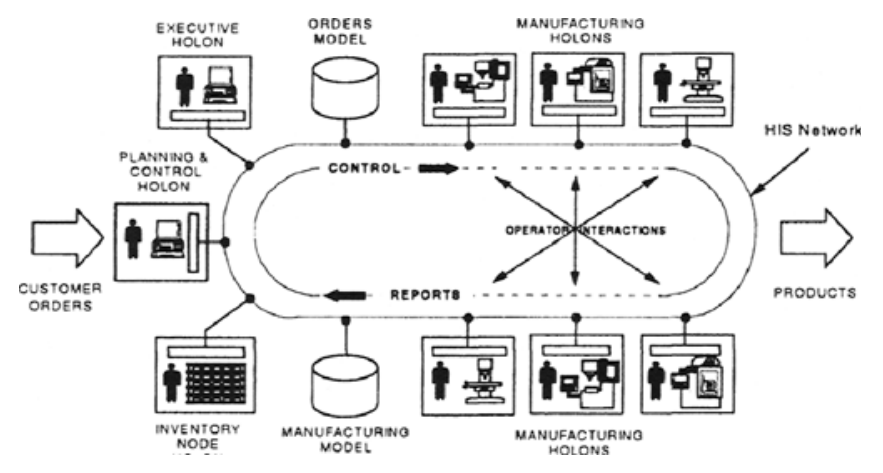

Figure 2 - Holonic Information System

\subsection{A Holonic Structure for Planning and Control of SMEs}

A heterarchical structure in which a collection of loosely coupled autonomous planning systems are co-operating to achieve an overall manufacturing goal is adopted to develope a planning and control solution tailored to the needs of SMEs, referred to as 'Distributed Autonomous Real Time' (DART) planning and control system (Rahimifard et al. 1999). DART utilise a simpler view of SME organisations which classifies these planning and control tasks into two categories of factory level and workstation level. Factory level tasks include the executive and business support activities, carried out by holons such as an executive holon, inventory holon and scheduling holon. The workstation level tasks represent the manufacturing activities, undertaken by manufacturing holons. The factory level tasks convert the customer orders into a set of production orders and the workstation level tasks 
execute tirsse production orders to generate the required products. In DART a realtime plannung approach is utilised based on generation of a 'production order list' (POL). Access to the POL is made available to both the planners and operators across the HIS network. The planner within the scheduling holon has the capability of forcing a production order onto a specific manufacturing holon, or allow them to be selected and processed by one of the manufacturing holons. At workstation level, a large number of criteria, such as priorities, due dates, slack times and commonality with present machine and tool set ups are taken into account to select and process a production order. The completion of the production order is then flagged, and appropriate information with regard to processing of the order is recorded.

\subsection{The Holonic Manufacturing Workstation}

A holonic manufacturing workstation in this research is considered as the coupling of the human operator with a manufacturing workstation, which has been enhanced through implementation of a specially designed IT support system to aid the operator for the effective operation of the workstation in an autonomous and cooperative manner. This is achieved through the design of a modular software structure consisting of mainly commercial manufacturing software tools which are integrated with other modules across the HIS. As stated, these software modules are grouped at each manufacturing holon to support :-

a) Autonomous Functions : These are specific to each and every workstation and are required for local control of the workstation. For example within the machining facilities of a typical SME, such functions could include the modifications of existing part programs, generation of inspection code, management of tools and fixture set-ups, work sequencing, and material planning. Such local functionality enables operators to utilise their skills more effectively in responding to changes in demands and increasing the overall performance of the workstation.

b) Co-operative Functions : These are related to the common business and manufacturing goals across the HIS network which are supported by individual operators such as updating shared information relating to customer orders, process planning, manufacturing operations, production planning and inventory control. In addition, co-operative functions enable the operator to view and communicate with other holonic nodes attached to the HIS, as depicted in figure 3.

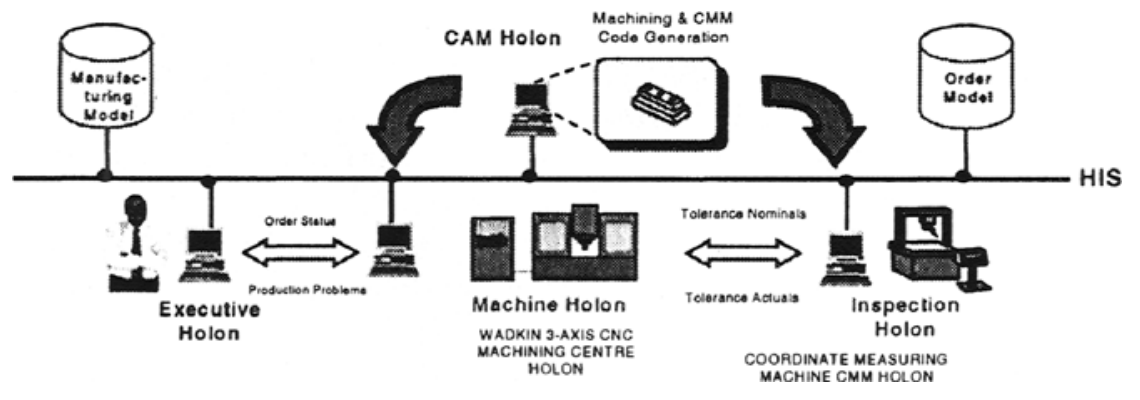

Figure 3 - Autonomous co-operative operations of holonic workstations within HIS 


\section{LABORATORY-BASED DEMONSTRATOR}

One of the main objectives of the research has been the development of visualisation and training tools to facilitate the wide adoption of unfamiliar and complex holonic concepts within contemporary industrial applications. Therefore, a comprehensive laboratory based demonstrator based on the configuration of a typical machining cell within a small metalworking enterprise has been developed, as shown in Figure 4. This production facility consists of a number of computer numerical control milling and turning machines, CNC vertical and horizontal machining centres, a range of conventional machine tools, a co-ordinate measuring machine, and a tool pre-setting workstation. The manufacturing facilities are linked together via a computer network (Microsoft NT network) and each area of manufacturing activity (a manufacturing holon) is equipped with a computer (running Windows NT 4.0). Each of these manufacturing holons is manned by one or more operators. It should be noted that such manufacturing holons could consist of a single workstation (e.g. a PC based CNC machining centre) or a collection of workstations grouped together around a computer node which is linked to the information network (e.g. conventional machine tools or tool prestters). In addition, a number of other computers within the design office, planning office, part and tool store and managers office are linked to this information network which represent the CAD/CAM holon, scheduling holon, inventory holon and executive holon (see figure 4). A special purpose user interface has been designed and implemented which is hosted by every computer linked to the network. This user interface not only provides access to all the software modules supported by the information network, namely the order database, the manufacturing database, the inventory module and the DART scheduler, but also enables the operators of various holons to execute a range of special purpose software packages which are required to support their daily functionality. In addition, this user interface supports a range of bi-directional messaging activities between the various holons within the system, so that not only the instructions can be downloaded by the executive holon or planning holon to the manufacturing holons, but also various types of queries (negotiations) can be made between each holon in the system. In this demonstrator, the typical functions supported by the machining centre holon are:-

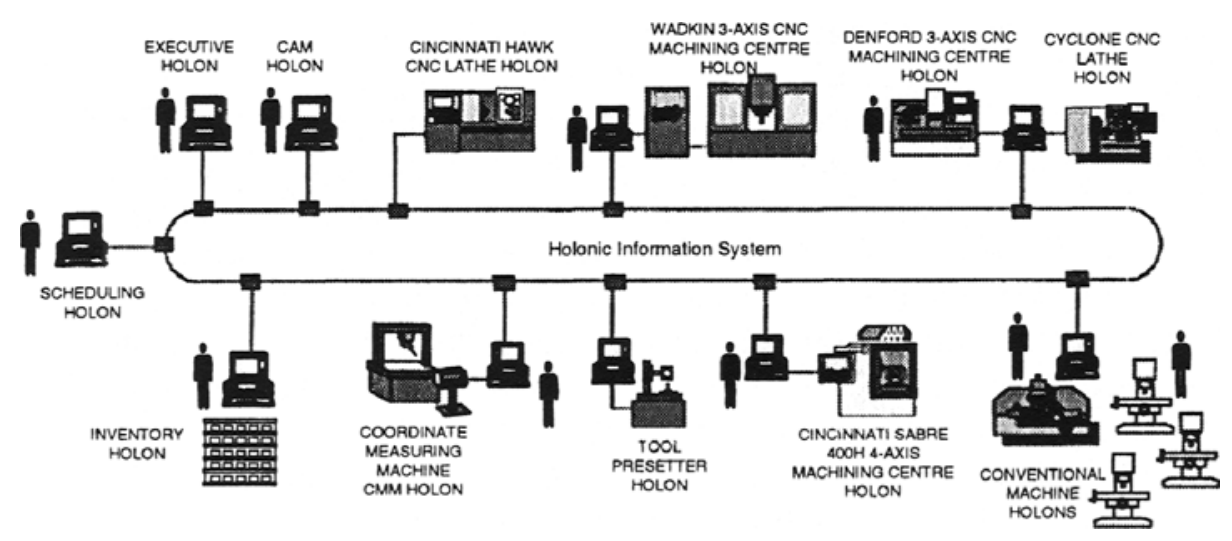

Figure 4 - Schematic of a Laboratory Based Demonstrator 
- NC Program Re-generation : enables operator to modify part programmes due to changes regarding the size of the raw material billet, tooling configurations, or even small alterations to the design of the component to be machined. There are several commercial systems for the purpose of regeneration of partprograms. One such system is the Mazak CAMWARE (MAZAK 2000) which provides functions such as minor design modifications, tool path simulation and machine code regeneration. Another important feature of this approach is the tool selection being based on the existing tool configuration with the machine tool magazine thus reducing the need for tool exchange and moving the tool to a different pocket number due to restrictions in a part program.

- NC Program Validation \& NC Machine Simulation : the importance of validation and simulation at the machining processes is very high, as any errors may potentially be very costly due to tool breakage and machine down times, wasted material and delays in delivery times. Therefore a commercial package called VERICUT (CGTech 1998) is used to verify modifications and give the operator confidence before the re-generated part programs are executed.

- Tool Management : is used to allow the operator to locate tools and fixtures, from their machine without having to physically go and search through various buffers and storage. Again, a commercial software package, namely ISISATMS (ISIS 2000) is used. This system is capable of informing the operator of the location of a tool item and if it is available for use, assigned to another machine, or actually in use. It can also provide the information concerning whether a particular tool is in a suitable state for the job, i.e. the level of wear it has incurred and whether it has enough life left. To this end, any tool can be swapped locally by the operator, within the workstation or between workstations, to maximise the potential of any set of tools.

- Material Planning : allows the operator to have visibility of the material flow and inventory control, and to change some aspects of the material allocated to a specific job e.g. billet size due to a temporary material shortages. A commercial MRP II package, namely Alliance / MFG (1999) has been incorporated within various holons in the HIS to support such functionality.

- Work Sequencing : the DART software has been developed using a commercial finite capacity planning system, called PREACTOR (Preactor International 2000). It consists of a number of PREACTOR modules that have been significantly reconfigured to generate a scheduling holon together with a number of local work sequencing modules hosted by each of the other holons within the HIS. This facility enables the workstation holon to receive work orders and instructions from the executive or scheduling holons, whilst at the same time permitting changes to be made locally to the work sequence. The operator may want to change the work sequence for a variety of reasons - from minimising the tool and fixture changes, to slotting in a high priority job.

- Local Performance Monitoring : is used to assess any deviations in performance of the machine tool, which may provide an early indication of a problem or fault with the machine. Typical monitoring issues are the accuracy of the machine tool and the set-up and cycle times which are recorded to allow analysis of the amount of time spent actually cutting material, and also the converse of this to view where the majority of non-machining time is spent. 


\section{CONCLUDING DISCUSSIONS}

In the contemporary global market, SMEs must consider the use of modern IT tools and networking to maximise productivity and responsiveness to customer orders. The requirements for such responsiveness to customer requirements have intensified in recent years, as manufacturing SMEs have experienced an urgency for greater flexibility to deal with highly individualistic customer desires for participation in the design and production procedures within the 'engineering-to-order' sector, and the unpredictable pattern of demands in the 'make-to-order' sector. In the late 1980's, and early 1990's, it was commonly assumed that the implementation of CIM concepts would provide such flexibility. This notion is now being challenged by the increasing belief that the implementation of CIM results in manufacturing systems which are in general too rigid and that the attributes of such highly integrated systems with totally predictable patterns of operation will not meet the needs of a modern SMEs.

In pursuit of this greater reactivity, there is a growing opinion that current CIM based organisations should be replaced by more innovative structures based on a conglomerate of distributed and autonomous units which operate as a set of cooperating entities, such as those defined by holonic concepts. This paper has illustrated the development of an IT facility which has been designed and implemented based on holonic concepts for the small manufacturing enterprise. However, the effective utilisation of such novel IT tools and working practices necessitates a significant transition from traditional methods. One of the major tasks facing the researchers of holonic concepts is convincing the industrial decision makers and strategists in particular those within SMEs that their operators can improve performance with such IT facilities and provide the desired flexibility and responsiveness to the customer demands. Identification of the most effective method for description and demonstration and of complex holonic concepts to busy production managers, engineers and operators with varying manufacturing and business knowledge and skills provides tough and interesting challenges. The laboratory-based demonstrator described in this paper is believed to provide a powerful visualisation facility to meet these challenges. Furthermore, this can be used as an efficient training tool for the operator of typical SMEs to familiarise themselves with the modern autonomous co-operative working environment, before adoption of such concepts within their daily operations. The author acknowledges the difficulties in introducing such significant changes in working cultures, but argues that the utilisation of such holonic structures and systems provide a number of commonly desired operational characteristics within SMEs, i.e.

- Reducing delays in processing of customer orders within the pre-production activities.

- Supporting distributed decision making to strengthen the role of the skilled and experienced production operators.

- Supporting frequent interactions between planners and operators via an information network.

- Incorporating a dynamic real time approach to enable extreme agility in responding to customer pressures. 


\section{ACKNOWLEDGEMENTS}

This work has been carried out as part of a collaborative research programme at Loughborough University and funded by the Engineering and Physical Science Research Council (EPSRC), entitled 'IT Tools to Improve Manufacturing Performance of Metalworking SMEs' (GR/L/27077). The author would also like to acknowledge the contributions Dr $\mathrm{N}$ Shires of PREACTOR International, $\mathrm{Mr} \mathrm{G}$ Herrington of ISIS Informatics Ltd and the colleagues of the Advanced Manufacturing Technology Centre at Loughborough University.

\section{REFERENCES}

1. Alliance Manufacturing Software International, 1999, Warrens Business Park, Leicester - UK, http://www.alliancenfg.com.

2. Balasubramanian S, Zhang $X$, Norrie DH, 2000, Intelligent control for holonic manufacturing systems, Proceedings of Institution Mechanical Engineers Part-B: Journal of Engineering Manufacture, 214 (B10), 953-961.

3. CGTech Ltd, 1999, The Coach House Shipwright's Yard, Brighton - UK, http://www.cgtech.com.

4. Gou L, Luh PB, Kyoya Y, 1998, Holonic manufacturing scheduling: architecture, co-operation mechanism and implementation, Computers in Industry, 37 (3), 213-231.

5. ISIS Informatics Ltd, 1999, Woodside Park, Godalming - UK, http://www.isistool.co.uk.

6. Koestler A, 1967, The Ghost in the Machine, (Arkana Books, London).

7. Leeuwen EH and Norrie D, 1997, Holons and Holarchies, Manufacturing Engineering, 76 (2), 8688.

8. Matthews J, 1995, Organisational foundations of intelligent manufacturing systems- the holonic viewpoint, Computer Integrated Manufacturing Systems, 8 (4), 237-243.

9. Preactor International, 2000, Cornbrash Park, Chippenham - UK, http://www.preactor.com.

10. Rahimifard S, Newman ST and Bell R, 1999, Distributed Autonomous Real Time Planning and Control of SMEs, Institute of Mechanical Engineer - Part B : Journal of Engineering Manufacture, 213 (B5), 475 - 489.

11. Shin J, Cho H, 2001, Planning and sequencing heuristics for featured-based control of holonic machining equipment, International Journal of Flexible Manufacturing Systems, 13 (1), 49-70.

12. Sousa P, Ramos C, 1999, A distributed architecture and negotiation protocal for scheduling in manufacturing systems, Computers in Industry, 38 (2), 103-113.

13. Suda H, 1990, Future Factory Systems Formulated in Japan, Japanese Journal of Advanced Automated Technology, 23 (3), 51-61.

14. Sugimura N, Moriwaki T, 1996, Modelling of holonic manufacturing system and its application to real-time scheduling, Proceedings of CIRP Seminars - Manufacturing Systems, 25 (4), 345352.

15. Tanaya PI, Detand J, Kruth J.-P., 1997, Holonic machine controller: a study and implementation of holonic behaviour to current NC controller, Computers in Industry, 33 (2-3), 323-333.

16. Toh KTK, Newman ST, Bell R, 1998, An Information Architecture for Small MetalWorking Companies, Proceedings of Institution Mechanical Engineers-Part-B: Journal of Engineering Manufacture, 212, 87-103

17. Toh KTK, Newman ST, Bell R, 1998, An Information Architecture for Small MetalWorking Companies, Proceedings of Institution Mechanical Engineers-Part-B: Journal of Engineering Manufacture, 212, 87-103.

18. Tonshoff HK, Winkler M, 1996, Shop control for holonic manufacturing systems, Proceedings of CIRP Seminars - Manufacturing Systems, 25 (3), 227-281.

19. Van Brussel H, Valckenaers P, Wyns J, Bongaerts L, Detand J, 1996, Holonic Manufacturing Systems and LiM, in Brown et. al. (Eds.) : IT and Manufacturing Partnerships, 185-196.

20. Wyns J, Van Brussel H, Valckenaers P, Bongaerts L, 1996, Workstation Architecture in holonic manufacturing systems, Proceedings of 28th CIRP International Seminar on Manufacturing Systems, Johannesburg, South Africa. 